\title{
Visualization of Wave Phenomena by an Array of Coupled Oscillators
}

\section{Dr. Günter Bischof, Joanneum University of Applied Sciences}

Throughout his career, Dr. Günter Bischof has combined his interest in science and engineering application. He studied physics at the University of Vienna, Austria, and acquired industry experience as development engineer at Siemens Corporation. Currently he teaches Engineering Mathematics at Joanneum University of Applied Sciences. His research interests focus on automotive engineering, materials physics, and on engineering education.

\section{Mr. Thomas Singraber B.Sc., Joanneum University of Applied Sciences}

Thomas Singraber obtained his B.Sc. in Automotive Engineering at the FH Joanneum, University of Applied Sciences Graz, Austria. Currently he is working on finalizing his Master's Thesis at the same faculty with a company partner supplying components to top motorsport teams all over the world. During his time at the Formula Student team he focused his work on aerodynamics and chassis developement and achieved therefore practical knowledge on a wide spectrum of racing topics. On completion of his studies, he intends to pursue an interdisciplinary career in the automotive sector with a strong motorsport affiliation.

\section{Mr. Christian J. Steinmann, HM\&S IT-Consulting}

Christian Steinmann has an engineer degree in mathematics from the Technical University Graz, where he focused on software quality and software development process assessment and improvement. He is manager of HM\&S IT-Consulting and provides services for SPiCE/ISO 15504 and CMMI for development as a SEI-certified instructor. He performed more than 100 process assessments in software development departments for different companies in the finance, insurance, research, automotive, and automation sector. Currently, his main occupation is a consulting project for process improvement for safety related embedded software development for an automobile manufacturer. On Fridays, he is teaching computer science introductory and programming courses at Joanneum University of Applied Sciences in Graz, Austria.

\section{Mr. Marton Szabo-Kass B.Sc., Joanneum University of Applied Sciences}

Marton Szabo-Kass obtained his B.Sc. degree in Automotive Engineering at Joanneum University of Applied Sciences in Graz, Austria, after which he is currently completing his M.Sc. studies at the same faculty. He gained practical experiences through leading the Formula Student racing team of his university and doing internships at high-tech automotive companies such as Porsche Motorsport. On completion of his studies, he intends to pursue an interdisciplinary career in the automotive and motorsport sector.

\section{Mr. Stefan Woerndl B.Sc., Joanneum University of Applied Sciences}

Stefan Woerndl obtained his B.Sc. degree in Automotive Engineering at the University of Applied Sciences Joanneum Graz. Currently he is completing his M.Sc. studies at the same faculty. Prior to this he gained some work experience as a technician, also in the automotive sector. On completion of his studies, he intends to pursue a career in research. 


\title{
Visualization of wave phenomena by an array of coupled oscillators
}

\begin{abstract}
Mechanical engineering curricula typically include courses in classical mechanics and continuum mechanics. Classical mechanics is governed by the Newtonian axioms, which lead to ordinary differential equations as the equations of motion. The mathematical description of continuum mechanics, on the other hand, is based on partial differential equations, describing the conservation laws and the constitutive relations. The underlying theories of ordinary and partially differential equations are usually covered in different mathematics courses, and a typical approach to a first discussion of partial differential equations in engineering mathematics is the heuristic derivation of the transversal wave equation of a vibrating string. Another approach, the continuum limit of the loaded string, leads to the one-dimensional longitudinal wave equation.
\end{abstract}

Both approaches start from the ordinary differential equations of Newtonian mechanics and lead to partial differential equations of continuum mechanics. The advantage of the continuum limit of a chain of masses connected by springs is that it is easily comprehensible for students and, in addition, many materials respond to small perturbations just as if they were a system of coupled oscillators. This harmonic oscillator response to perturbations leads in a continuum model to the appearance of wave phenomena.

For the visualization of such wave phenomena, a computer program that simulates a twodimensional spring-mass system has been developed within an undergraduate student project. The model consists of a rectangular lattice of regularly spaced point masses connected to each other and to the rigid boundary by a network of massless springs. The force on each mass is computed due to its spring connections with its neighbors, along with external forces such as gravity. Energy dissipation can be added to the model on demand by viscoelastic damping. The motion of each particle is governed by Newton's second law, which requires the solution of a system of coupled ordinary differential equations. This is done in the C\# programming language via a variety of implemented numerical integration schemes. The computer program allows the visualization of the motion of the point masses, which can be initiated by the displacement of an arbitrary number of masses via mouse drag.

The motion of a single mass connected by springs to the adjacent walls closely resembles the behavior of the well-known harmonic oscillator. The introduction of additional masses brings particle interaction into effect, which leads to energy transfer in the system. When the number of involved mass points is increased, the moving particles increasingly appear to the observer as a continuous system. This is due to a scale change that links the microstructure of coupled particles with the macroscopic behavior of a continuous material, which includes wave behavior like interference effects. The dynamic visual output of the program can increase and enhance understanding of various wave phenomena and is therefore well suited as both a teaching aid and an analysis tool. 


\section{Introduction}

The curriculum of the undergraduate Automotive Engineering degree program at Joanneum University of Applied Sciences includes Engineering Mathematics in the first three semesters. The lectures follow typically the contents of text books like Kreyszig's Advanced Engineering Mathematics ${ }^{1}$, with an emphasis on ordinary differential equations in the second and partial differential equations in the third semester. In both semesters analytical as well as numerical methods for the solution of differential equations are discussed in order to give the students an idea of how real world problems can be solved.

The mathematics course curriculum provides a guideline on a logical succession of engineering courses. Classical mechanics, which is dealing with the equilibrium and the motion of particles and systems of particles, is based on ordinary differential equations. The dynamics courses are therefore taken by our students not earlier than in the second semester of study. Continuum theories - which include fluid mechanics and elasticity - are governed by partial, rather than ordinary, differential equations, which make them accessible only to third semester or higher level students.

Although partial differential equations provide elegant continuous models it should be made clear to the students that they build only an approximation of fundamentally discrete phenomena. For example, a vibrating string is usually regarded as a continuous object, yet if one looks at a fine enough scale, the string is made up of atoms, suggesting a discrete model with an incredibly huge number of degrees of freedom. However, the modelling of macroscopic systems with a simple bottom-up-from-the-atoms strategy is currently out of reach and will require major breakthroughs in computational mathematics at multiple scales ${ }^{2}$. The problem of bridging large disparities in spatial and temporal scales, also known as the tyranny of scales ${ }^{3}$, dominates simulation efforts in nanoscience, molecular modeling of biological systems, advanced materials, turbulence modeling, and many other applications. These problems involve a very broad range of scales amenable to a single description, such as continuum theory in the case of turbulence.

Nevertheless, continuum model equations such as the equations for elastic solids work despite the fact that they almost completely ignore small scale or atomistic details. In most engineering applications materials display linear elasticity; they obey Hooke's law. One phenomenological parameter related to its stiffness properties is Young's modulus appearing in the equations of motion for solids as well as in equilibrium equations. At millimeter scales solids with isotropic behavior like construction steel or aluminum appear to be almost completely homogeneous. For behaviors that take place within this range of scale, such materials are well-modeled by equations that make only use of two parameters (bulk modulus and shear modulus). When performing a scale change from the large scale picture to its smallest atomic scale, typical materials for engineering purposes exhibit highly symmetric crystalline lattice structures. Construction steel at the macroscales, on the other hand, exhibits no crystalline structure. Somewhere between the lowest scale of symmetric crystals and the scale of millimeters the symmetric structures must disappear, which would suggest that properties of the materials at their most basic, atomic level do not determine the properties of the materials at macroscales ${ }^{3}$.

In reality, the story is in fact much more complex due to the appearance of symmetry breaking structures like vacancies, dislocations and wall defects in intermediate, "mesoscopic" scales. 
For the description of the behavior of macroscopic ("continuous") systems we have, in principle, the choice between bottom-up and top-down modeling. In the case of a vibrating string or a vibrating membrane built up of particles (atoms or molecules), the bottom-up modelling from the microscale is accomplished by starting with a general dynamical principle such as Newton's second law. Depending on the class of material, the forces acting between the particles have to be determined, which are assumed as linear elastic in the case of vibrating springs and membranes. The sum of forces acting on each particle has to be set equal to $m_{i} \ddot{\mathbf{x}}_{i}$ to yield the equation of motion of the $i^{\text {th }}$ particle. This yields an incredibly huge coupled system of ordinary differential equations that should be solved in order to understand the behavior of the macroscopic system. In most cases, further simplifications employing variable reduction and averaging are necessary to gain the desired information.

In the top-down formulation of the equations of motion of elastic bodies the fundamental equations of continuum mechanics are employed. At macroscales, forces within a continuum can be represented by the second-rank Cauchy stress tensor $\sigma$, and the equation of motion can be expressed as $\nabla \cdot \sigma+\mathbf{F}=\rho \ddot{\mathbf{u}}$. For isotropic linear elastic solids, symmetry considerations come into play and lead to the Navier-Cauchy equation with the two Lamè parameters that express the empirical details about the material response to strain. These continuum model equations work despite the fact that they completely ignore small scales or atomistic details.

The question arises why the continuum models work on the macroscale, and what is the relationship between the (bottom-up) dynamical models that track the behavior of individual atoms and molecules, and (top-down) continuum models like those of Navier-Stokes and Cauchy that are applicable at the scale of millimeters and above. Can the continuum account of the vibration behavior of an elastic body (i.e. a string or membrane in this paper) be reduced to the theory of its atomic constituents in the sense that we can derive the macroscale behavior from the microscale (particle) theory?

The upscaling of the model descriptions from atomic to laboratory scales usually takes place by reduction. The individual description of elastically coupled particles reduces to continuum mechanics by making use of appropriate mathematical limits, which seems to be justified by mathematical convenience and empirical adequacy ${ }^{4}$. This applies, in particular, to the many classical continuum models of fluids and solids that are obtained by taking a limit of a classical atomistic model as the number of atoms $N$ tends to infinity in an appropriate way, e.g. by keeping the mass density constant. The mathematics of infinity is much more convenient as the mathematics of the large finite. In the spatial limit edge and boundary effects are absent in an infinitely large system; temporal limits, on the other hand, extinguish transient effects. Continuum modeling on this view represents an idealization, "a smoothedout imitation of a really much more complicated microscopic world." 5

The recipe by which continuum models are constructed is quite clear and leads in many cases to equations that successfully characterize the behavior of systems at the macroscopic level. When, however, it comes to the solution of the partial differential equations obtained in the continuum limit, the students are often puzzled by the fact that the problem has to be discretized again in order to solve it numerically.

A comprehensive guidance through the interplay between continuous and discrete modelling is provided by Gilbert Strang's Introduction to Applied Mathematics ${ }^{6}$, which illuminates the natural correspondence between solution methods for systems of equations in discrete and 
continuous settings. It shows the common threads that all continuous and discrete problems share and how they translate into each other.

In order to engage our students with this tension between the discrete and continuous an interdisciplinary (mathematics, mechanics, and programming) student research project was initiated, which encompassed the development of a computer program for the simulation and visualization of a two-dimensional array of coupled oscillators. An added benefit of this program is that the visualization of vibrations in space and time can increase and enhance understanding of various vibration phenomena that are commonly difficult to conceptualize ${ }^{7}$.

The course Information Systems and Programming in the second semester of the degree program formed the basis of this project. In this course the programming language $\mathrm{C \#}$ is introduced. It is an object-oriented cross-platform programming language of the $\mathrm{C}$ family that enables the students to develop graphical user interfaces with comparatively little effort. The students are offered, complementary to this course, a variety of project proposals at the beginning of the semester. Usually a team of three works on such a project, with one student being designated as project leader. This structure promotes the development of certain generic skills, like the ability to work in teams, to keep records and to meet deadlines. The teams choose their projects according to their interests and skills. An important factor contributing to the acceptance and success is the usefulness and applicability of the projects. Usually two or more groups are assigned with the same task. In this way competition is generated, which in turn increases the students' motivation ${ }^{8,9}$.

Three first-year student teams took up the challenge to simulate and visualize the motion of material particles that are regularly arranged in a plane and coupled to each other and to the boundaries by elastic forces. The outcome of one of those projects is in the focus of this paper.

The remainder of this paper is structured by first providing a brief introduction to the dynamics of a mass spring system and its continuum limit. Secondly, there is a description of the computer program for the simulation and visualization of mass spring systems implemented by our students. Then this program is exemplified by the simulation of wave phenomena that can appear when the complexity of the discrete system exceeds a certain limit, and a summary then closes the paper.

\section{Mass spring system and continuum limit}

To better understand the physics of such a system it seems to be convenient to employ a onedimensional system consisting of particles aligned in a straight line. The model can be imagined as beads mounted on a thin wire and connected to neighboring beads by springs (Figure 1).

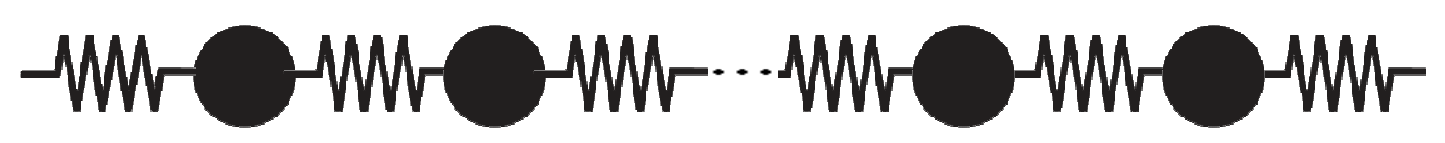

Figure 1: Schematic diagram of a one-dimensional coupled system of masses and springs.

The particles have the masses $m_{i}$ and all the springs connecting the particles shall have equal spring constants $k$. If the equilibrium position of the $i^{\text {th }}$ particle is $x_{i}$, then its position when disturbed from equilibrium can be denoted $x_{\mathrm{i}}+u_{i}$. In other words, $\mathrm{u}_{i}(t)$ is the displacement from equilibrium of the $i^{\text {th }}$ particle. When the particles have a constant equilibrium spacing $a$ 
then the distance between particle $i$ and particle $i+1$ is $a+u_{i+1}-u_{i}$. So the stretch of the spring is $\left(u_{i+1}-u_{i}\right)$ and the potential energy associated with the spring is proportional to its square. The Lagrangian of a system of $N$ elastically connected particles can therefore be written as

$$
L=T-V=\sum_{i=1}^{N}\left(\frac{1}{2} m_{i}\left(\frac{\mathrm{d} u_{i}(t)}{\mathrm{d} t}\right)^{2}-\frac{1}{2} k\left(u_{i+1}(t)-u_{i}(t)\right)^{2}\right) .
$$

The Euler-Lagrange equation for the $i^{\text {th }}$ particle is

$$
\frac{\mathrm{d}}{\mathrm{d} t} \frac{\partial L}{\partial \dot{u}_{i}}-\frac{\partial L}{\partial u_{i}}=0
$$

from which the following coupled equations of motion of the particles are derived:

$$
\left(\begin{array}{ccccc}
m_{1} & 0 & 0 & \ldots & 0 \\
0 & m_{2} & 0 & \ldots & 0 \\
0 & 0 & m_{3} & \ldots & 0 \\
\vdots & \vdots & \vdots & \ddots & \vdots \\
0 & 0 & 0 & \ldots & m_{N}
\end{array}\right)\left(\begin{array}{c}
\ddot{u}_{1} \\
\ddot{u}_{2} \\
\ddot{u}_{3} \\
\vdots \\
\ddot{u}_{N}
\end{array}\right)+\left(\begin{array}{ccccc}
-2 k & k & 0 & \ldots & 0 \\
k & -2 k & k & \ldots & 0 \\
0 & k & -2 k & \ldots & 0 \\
\vdots & \vdots & \vdots & \ddots & \vdots \\
0 & 0 & 0 & \ldots & -2 k
\end{array}\right)\left(\begin{array}{c}
u_{1} \\
u_{2} \\
u_{3} \\
\vdots \\
u_{N}
\end{array}\right)=\left(\begin{array}{c}
0 \\
0 \\
0 \\
\vdots \\
0
\end{array}\right)
$$

In this derivation fixed-wall boundary conditions $\left(u_{0}=u_{N+1}=0\right)$ have been assumed.

Equation (3) represents a coupled system of linear ordinary second-order differential equations, which can - in principle - be solved analytically, because the mass matrix and the stiffness matrix can be simultaneously diagonalized. This is no longer possible if dispersion (viscous damping) is introduced into the system; in that case the system has to be expanded into a $2 N \times 2 N$ coupled system of first-order differential equations (phase space approach). The stiffness matrix resembles a second difference matrix, which corresponds to the second derivative in the continuum limit.

The continuum limit of a chain of elastically coupled particles can be derived by first multiplying and dividing equation (1) by $a$, and letting $N \rightarrow \infty, a \rightarrow 0$, and by keeping the length of the chain $L_{0}=N a$ fixed (see e.g. Hamill ${ }^{10}$ ). The displacements $u_{\mathrm{i}}(t)$ transform into $u(x=i a, t)$ in which case

$$
T=\frac{1}{2} \sum_{i=1}^{N} \dot{u}_{i}^{2} \rightarrow \frac{1}{2} \int_{x_{1}}^{x_{2}} \lambda(x)\left(\frac{\partial u(x, t)}{\partial t}\right)^{2} \mathrm{~d} x
$$

with $\lambda(x)$ the linear mass density at location $x$, and

$$
V=\frac{1}{2} k \sum_{i=1}^{N}\left(u_{i+1}-u_{i}\right)^{2} \rightarrow \frac{1}{2} k \int_{x_{1}}^{x_{2}}\left(\frac{u(x+a, t)-u(x, t)}{a}\right)^{2} a \mathrm{~d} x
$$

Recognizing the spatial derivative in equation (5), one finally obtains the Lagrangian 


$$
L=\int_{x_{1}}^{x_{2}} \mathcal{L}\left(u, \partial_{x} u, \partial_{t} u\right) \mathrm{d} x
$$

with the Lagrangian density

$$
\mathcal{L}=\frac{1}{2} \lambda(x)\left(\frac{\partial u(x, t)}{\partial t}\right)^{2}-\frac{1}{2} K\left(\frac{\partial u(x, t)}{\partial x}\right)^{2}
$$

In the continuum limit, $K=k a$ now represents Young's modulus (for a proper limit, $\lambda$ and $K$ are neither infinite nor infinitesimal).

The action $S[u(x, t)]$ is

$$
S[u(x, t)]=\int_{t_{1}}^{t_{2}} \mathrm{~d} t \int_{x_{1}}^{x_{2}} \mathrm{~d} x \mathcal{L}\left(u, \partial_{x} u, \partial_{t} u\right)
$$

where $t_{1}, t_{2}, x_{1}$, and $x_{2}$ are the limits on the time and space coordinates, respectively. Setting $\delta S=0$ gives the Euler-Lagrange equation

$$
\frac{\partial}{\partial t}\left(\frac{\partial \mathcal{L}}{\partial\left(\partial_{t} u\right)}\right)+\frac{\partial}{\partial x}\left(\frac{\partial \mathcal{L}}{\partial\left(\partial_{x} u\right)}\right)-\frac{\partial \mathcal{L}}{\partial u}=0 .
$$

This yields the one-dimensional wave equation

$$
\frac{\partial^{2} u(x, t)}{\partial x^{2}}=\frac{1}{c^{2}} \frac{\partial^{2} u(x, t)}{\partial t^{2}}
$$

a fundamental example of a hyperbolic partial differential equation, where $c=\sqrt{K / \lambda}$ is the speed of wave propagation. The generalization of the above derivation to two and more dimensions is straightforward.

\section{Implementation}

The computer program written and implemented by the students in C\# is structured in the four modules User Operation, Calculation, Visualization, and Error Handling. An essential feature is the declaration of the class Particle, because classes support inheritance, a fundamental characteristic of object-oriented programming. Particle is assigned a number of properties, which are the mass of the particle, its position in the array, its velocity, and the force acting on the particle.

When the user defines the size of the two-dimensional coupled mass spring network, the program creates an array of particles and assigns the corresponding properties. The program distinguishes between internal particles and those which are on the boundaries and anchored to the "walls" (mechanical ground, i.e. Dirichlet boundary conditions). In Figure 2 the program's graphical user interface is illustrated. An array of 96 particles connected by springs to their nearest neighbors and to the walls has been generated. 


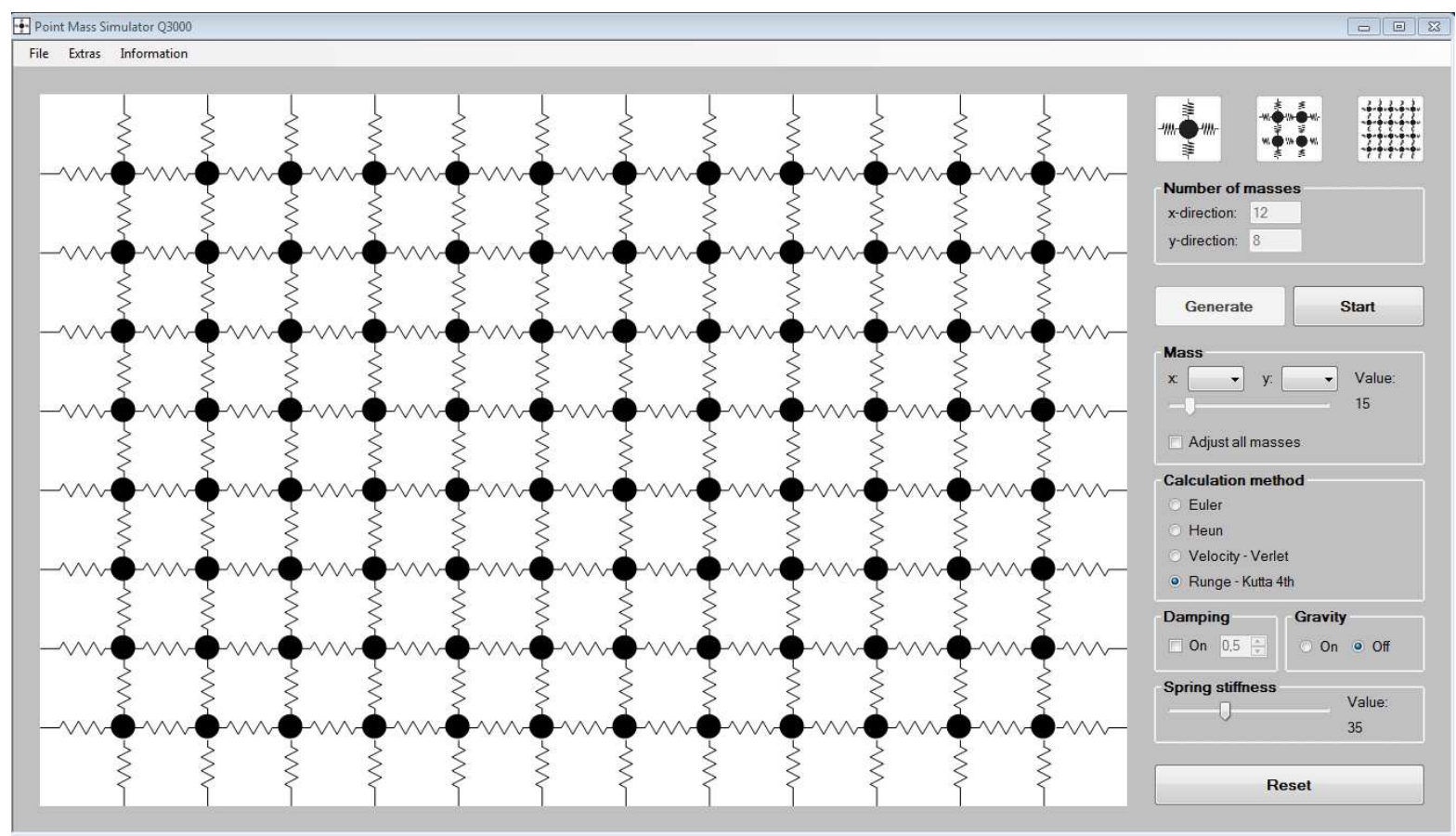

Figure 2: Graphical user interface of the program. An array consisting of $12 \times 8$ particles elastically connected to their nearest neighbors and to the boundaries has been generated.

The net force acting on each particle is then computed by summing the spring forces acting on the particle with any other external force, like gravity. The elastic force between the $i^{\text {th }}$ and $j^{\text {th }}$ particle is in principle given by

$$
\mathbf{F}_{i j}(t)=k_{i j}\left(\left|\mathbf{r}_{j}(t)-\mathbf{r}_{i}(t)\right|-l_{i j}\right) \frac{\mathbf{r}_{j}(t)-\mathbf{r}_{i}(t)}{\left|\mathbf{r}_{j}(t)-\mathbf{r}_{i}(t)\right|},
$$

with $\mathbf{r}_{i}(t)=\left(x_{i}(t), y_{i}(t)\right)$ being the position of the $i^{\text {th }}$ particle, $l_{\mathrm{ij}}$ the equilibrium distance between the respective particles and $k_{i j}$ the spring stiffness. In this program the equilibrium distances are constant in each direction and the spring stiffnesses are all equal $\left(k_{i j}=k\right)$.

The equation of motion for each particle is then given by

$$
\frac{\mathrm{d}^{2} \mathbf{r}_{i}(t)}{\mathrm{d} t^{2}}=\frac{1}{m_{i}} \sum_{j=1}^{4} \mathbf{F}_{i j}(t)
$$

The particles are moved according to the results obtained from the numerical integration of their equations of motion (12), and their associated springs are correspondingly stretched and compressed. For the visualization of the springs a Method has been programmed, that calculates their deformation accordingly (see Figure 3).

For the integration of equation (12) four different numerical methods have been implemented. By default the explicit Euler method is offered, but the user can switch by mouse click to Heun's predictor-corrector algorithm, to the Velocity-Verlet or to the fourth-order RungeKutta method. Thus, different orders of accuracy can be tested and compared for the same time step size. 

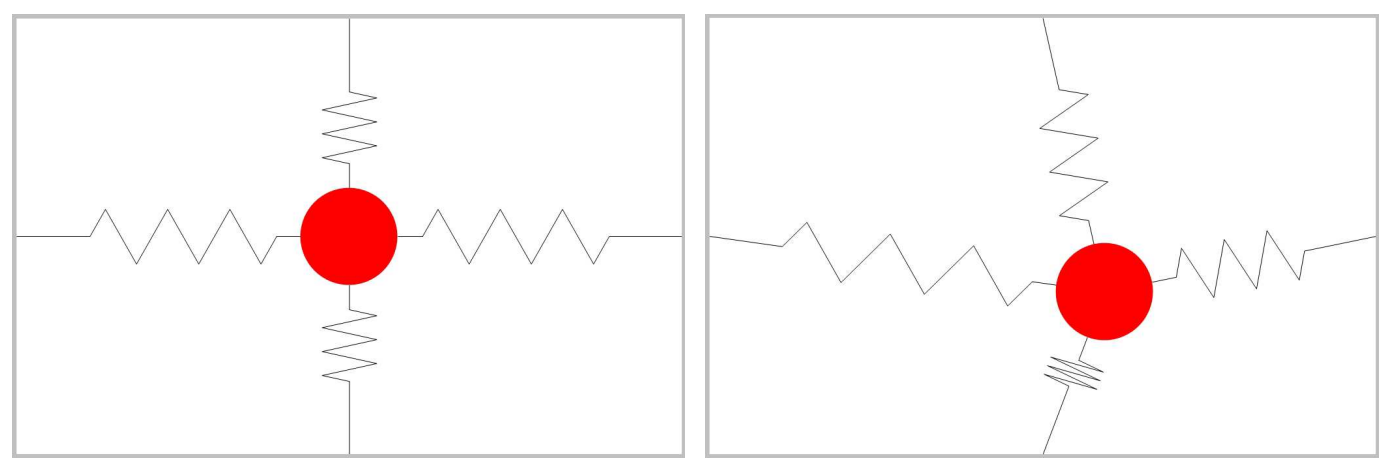

Figure 3: Single particle elastically attached to the boundaries (left: equilibrium position, right: deflected position).

Collision detection has not been implemented, which allows unphysical solutions in the case of large displacements. Nevertheless, large displacements should be avoided in any case for reasonable solutions in order to not violate the assumption of linear elasticity.

For the initial configuration of the coupled mass spring system three buttons are offered, i.e., single particle, four particles arranged in a square on a plane, and an arbitrary number of rectangularly arranged particles (see Figure 2). The spring stiffnesses are the same for all the springs and can collectively be adjusted by a slider. The default value is $35 \%$ of a predefined maximum stiffness. The masses of the particles in the array can be individually altered in accordance to equation (1). When the particle is clicked on by the mouse its color changes from black to red. Then its mass can be adjusted within a given interval by a slider, which is indicated by an increase or decrease of the particle's diameter. The displacement of the particles is also done by mouse. The particles can be individually shifted by left mouse clicking and dragging.

In Figure 4 five particles are illustrated that are elastically attached in a line to each other and to the boundaries. In this configuration it is possible to initiate pure longitudinal or pure transversal waves. As soon as a second layer of particles is involved both longitudinal and transversal waves are produced (Figure 5).
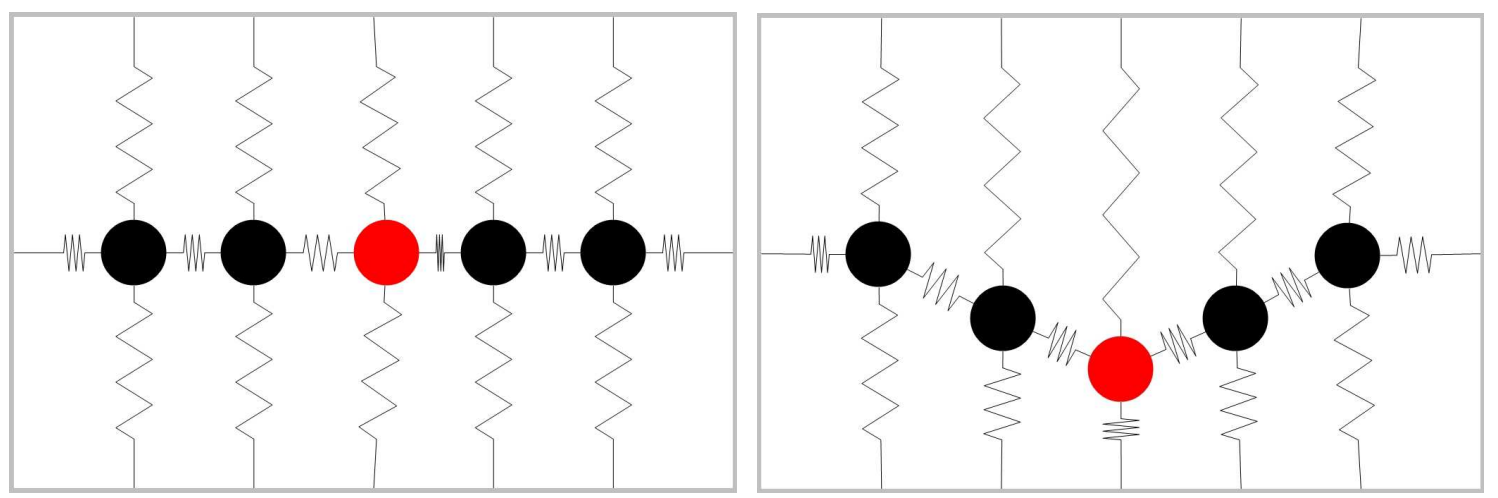

Figure 4: Five particles elastically attached in a line to each other and to the boundaries. In the left picture longitudinal oscillations are initiated, in the right picture a transversal wave will develop.

When a particle is pushed to a distance from its neutral position, the disturbance is transmitted to the adjacent particles by the springs, which creates a chain reaction (Figures 4 to 6 ). 


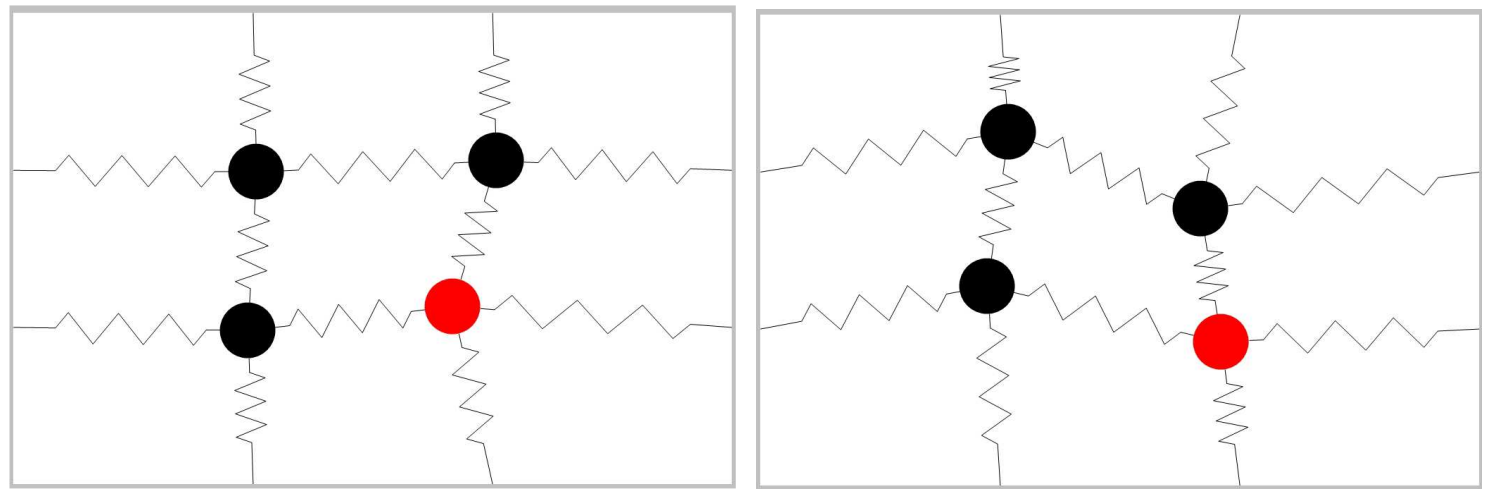

Figure 5: Four particles elastically attached to each other and to the boundaries. In the left picture only the highlighted particle is displaced. The right picture shows the dynamical reaction of the system.
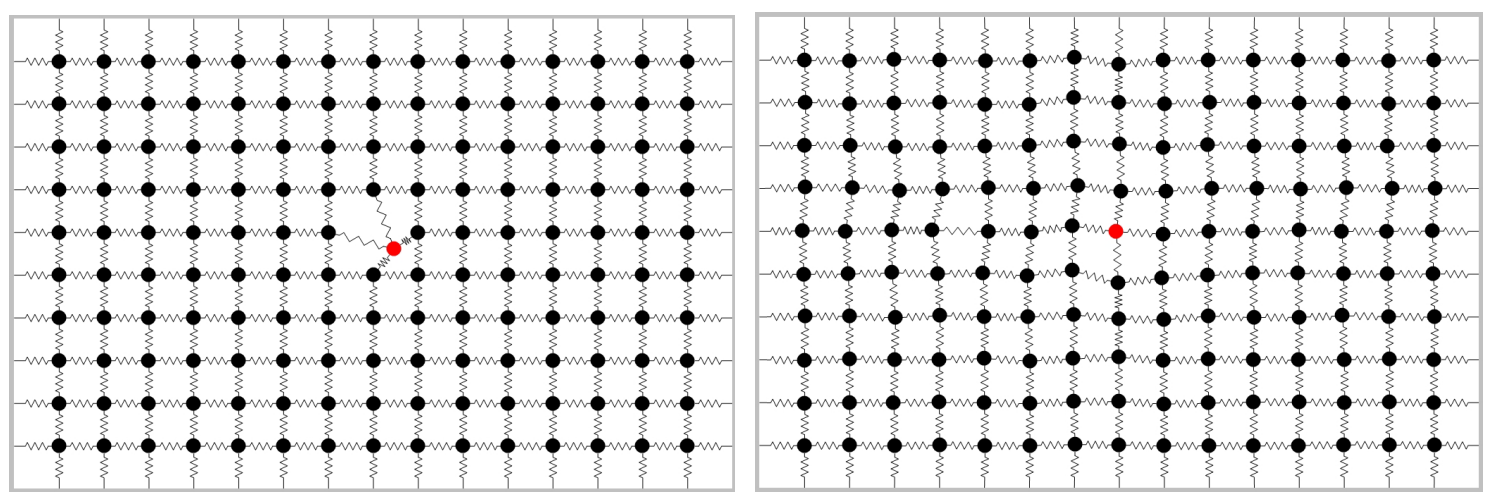

Figure 6: A particle in the centre of a $15 \times 10$ particle array is pushed out of its equilibrium position (left). In the right picture the disturbance has been transmitted to the adjacent particles by the springs.

In Figure 7 a tessellated mass distribution resembling the structure of an ionic crystal is shown. Moderate displacements of the heavier particles lead to more vigorous oscillations of the other sublattice.

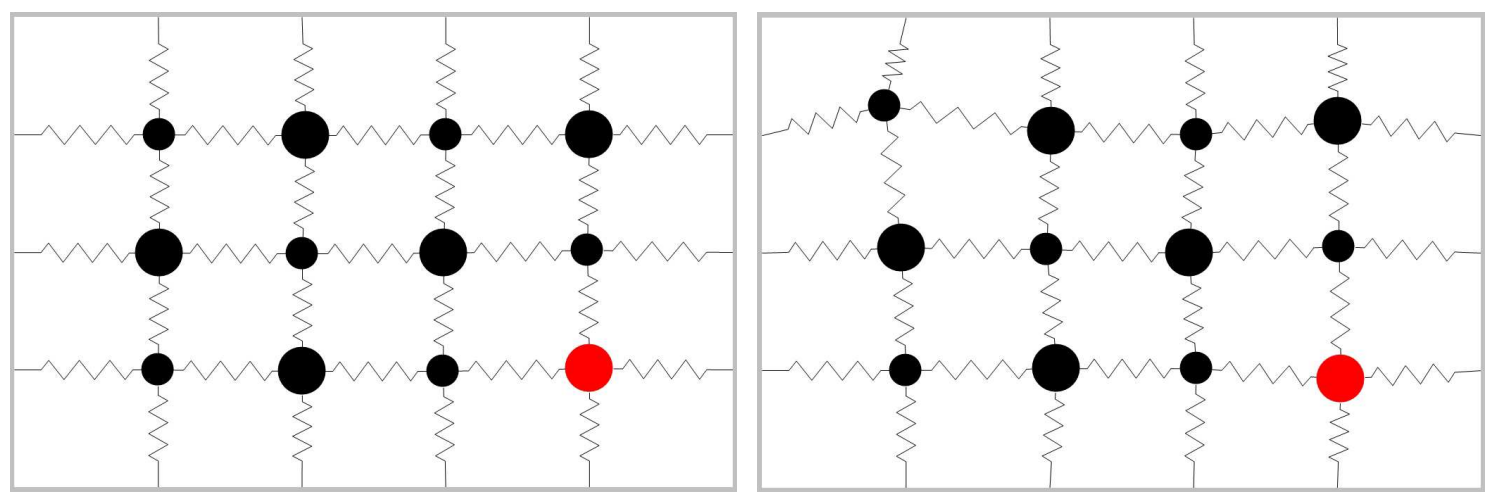

Figure 7: Array configuration resembling a two-dimensional ionic crystal structure

An external force field can be switched on that resembles gravity and acts in downward direction (Figure 8). To enable the mass spring system to reach an equilibrium configuration, viscous damping is added to the equations of motion (3) and can be activated via a check box. The damping constant is the same for all the springs and can be varied by a scroll bar.

The equilibrium configuration of the mass spring system in the gravitational field shows the expected catenary curves (Figure 8 ). 

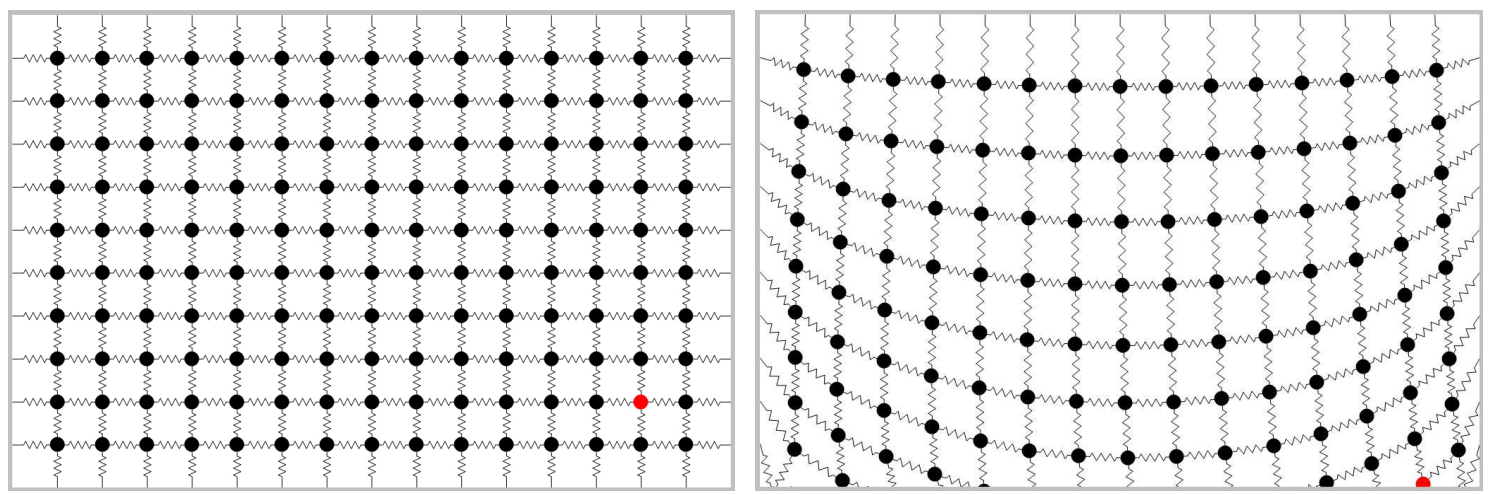

Figure 8: External field (gravity) acting on an array of 150 particles (left: equilibrium position without external field, right: equilibrium position in gravitational field).

When the size of the mass spring system exceeds $25 \times 20$ particles the plotting of the springs should be omitted, which can be specified in the system settings. The size of the particles can be minimized to a radius of one pixel, which is increased by a factor of three if the particle is selected by mouse click or by its coordinates. Its color is then also changed to red for a better detectability (see Figure 9).

In the case of larger systems it is convenient to store predefined configurations of initial displacements and mass distributions, which is also implemented in the program. Such systems require substantial computational effort, which slows down its dynamic visualization. For this reason an automatic storage routine is additionally implemented in the program that stores the configuration of the mass spring system after each calculational time step. The stored pictures can then be animated by free video tools like VirtualDub and processed by e.g. MeGUI.

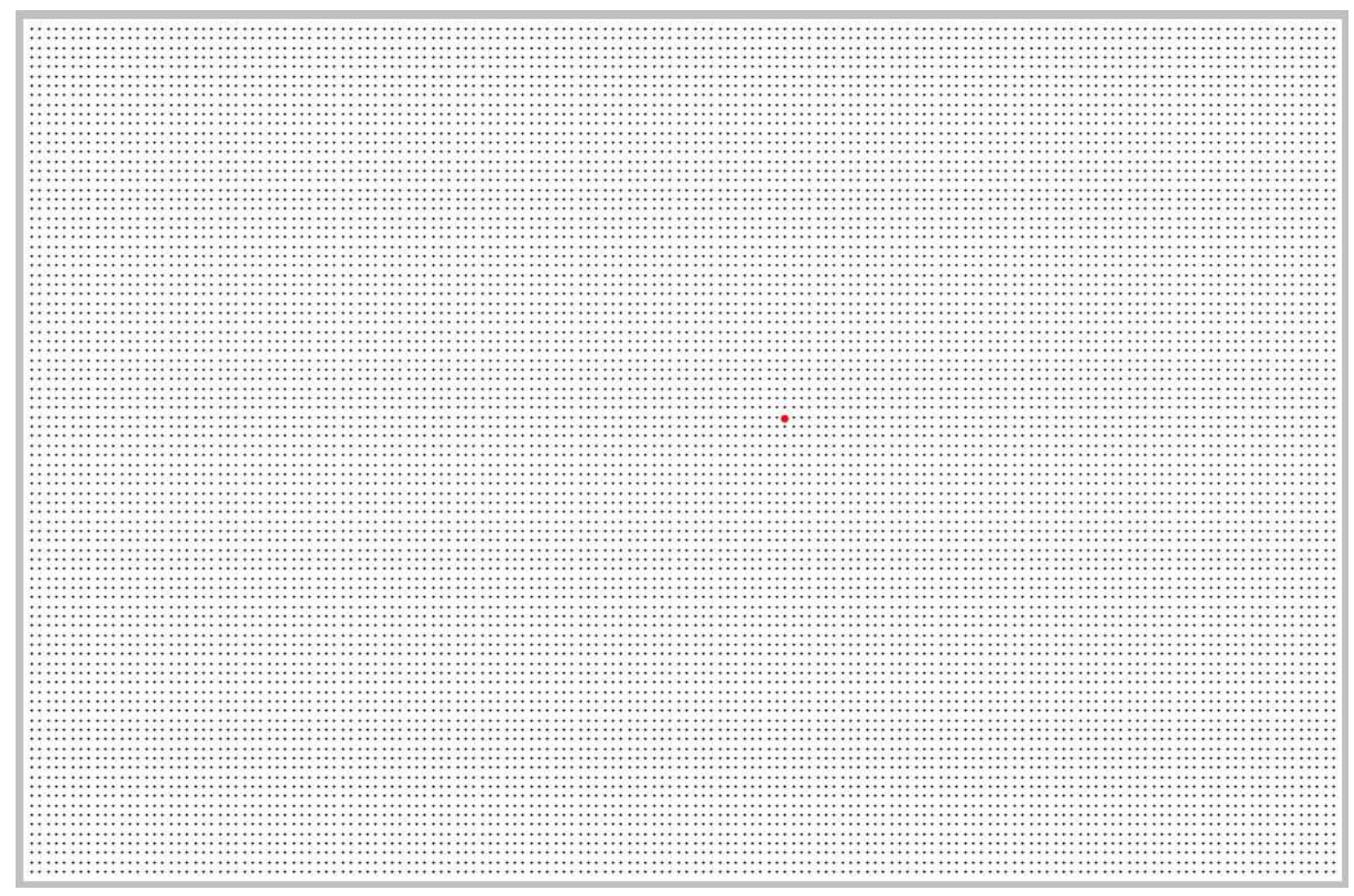

Figure 9: Mass spring system of $150 \times 90$ particles. The particles are set to unit size and the springs are not plotted. One particle has been highlighted by mouseclick for further settings. 


\section{Approximation of a continuous system}

As described above, the behavior of a macroscopic system can be approximated by taking a limit in which the inter-particle spacing approaches zero while the number of oscillators becomes arbitrarily large (the limits in equations 4 and 5). This approximation is applicable as long as the length scales associated with the macroscopic behavior of a material are much larger than the length scales associated with the microscopic structure, i.e. the inter-particle spacing ${ }^{11}$.

In an attempt to approach and visualize the continuum limit of a rectangular array of elastically coupled particles, a system of over 260000 particles with equal masses and spring constants was created. As initial condition, a particle in the middle of the left boundary and another particle in the middle of the right boundary were simultaneously displaced in opposite directions. Then the simulation was started within the highest-order integration scheme and the positions of the particles were stored after each time step. From these data a movie was created as described above. In the following figures (Figures 10 to 14) snapshots are shown, which were taken at different evolutionary phases of wave phenomena that occurred in the course of the numerical simulation.

The snapshot in Figure 10 shows two wave fronts that have already established and propagate towards each other. Both regions confined by the wave fronts show already a rich internal structure that is caused by several reflections at the boundaries (Dirichlet boundary conditions produce fixed end wave reflections) and by interference.

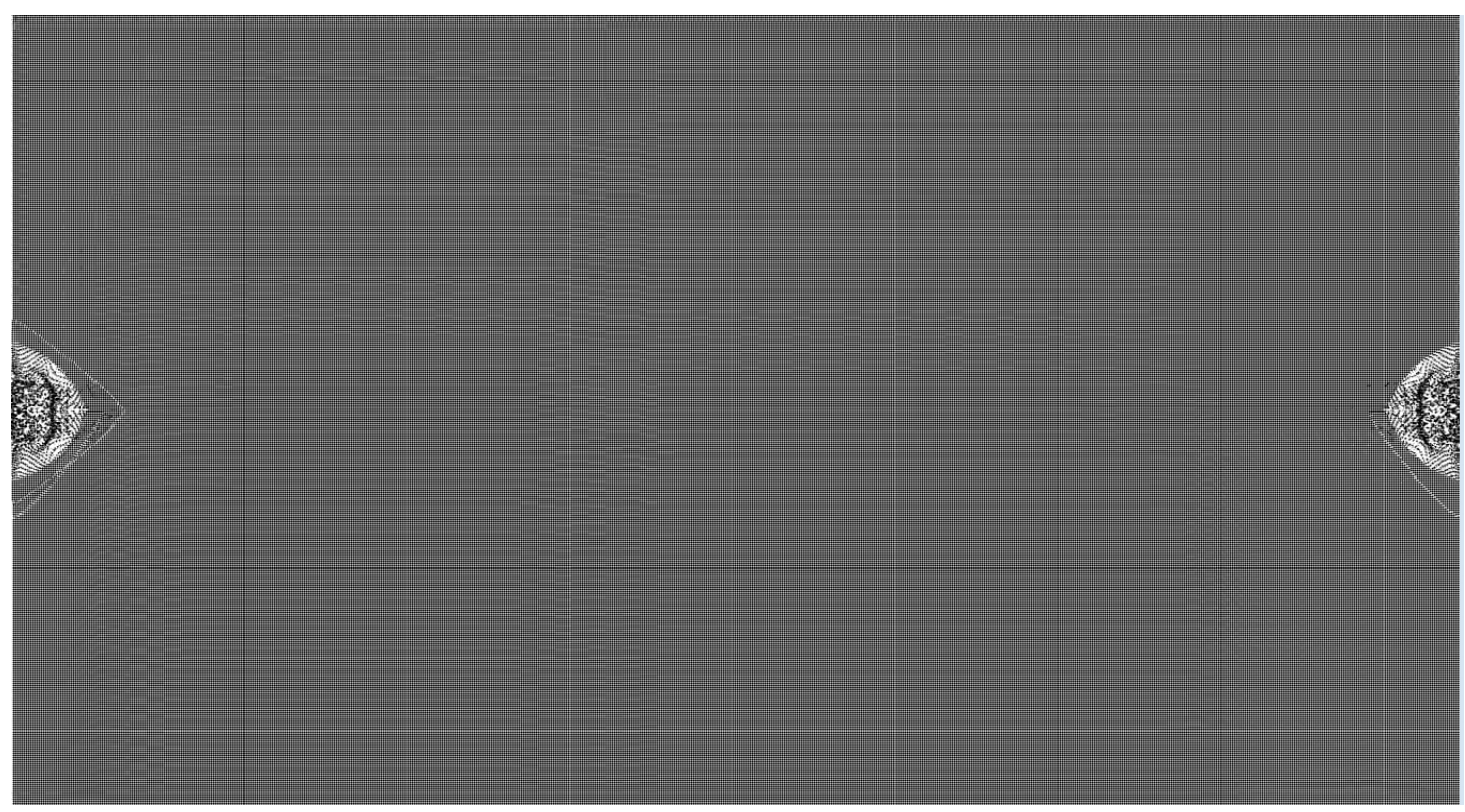

Figure 10: Array of 261206 particles $(682 \times 383)$. The initial condition was a simultaneous displacement of one particle in the middle of the left and of the right boundary, respectively. Two wave fronts have established and propagate towards each other.

In Figure 11 the wave fronts are close to contact but still not overlapping. Due to the fact that reflections now also occur at the upper and lower boundary, dark interference patterns with symmetric peaks in diagonal direction are formed. 


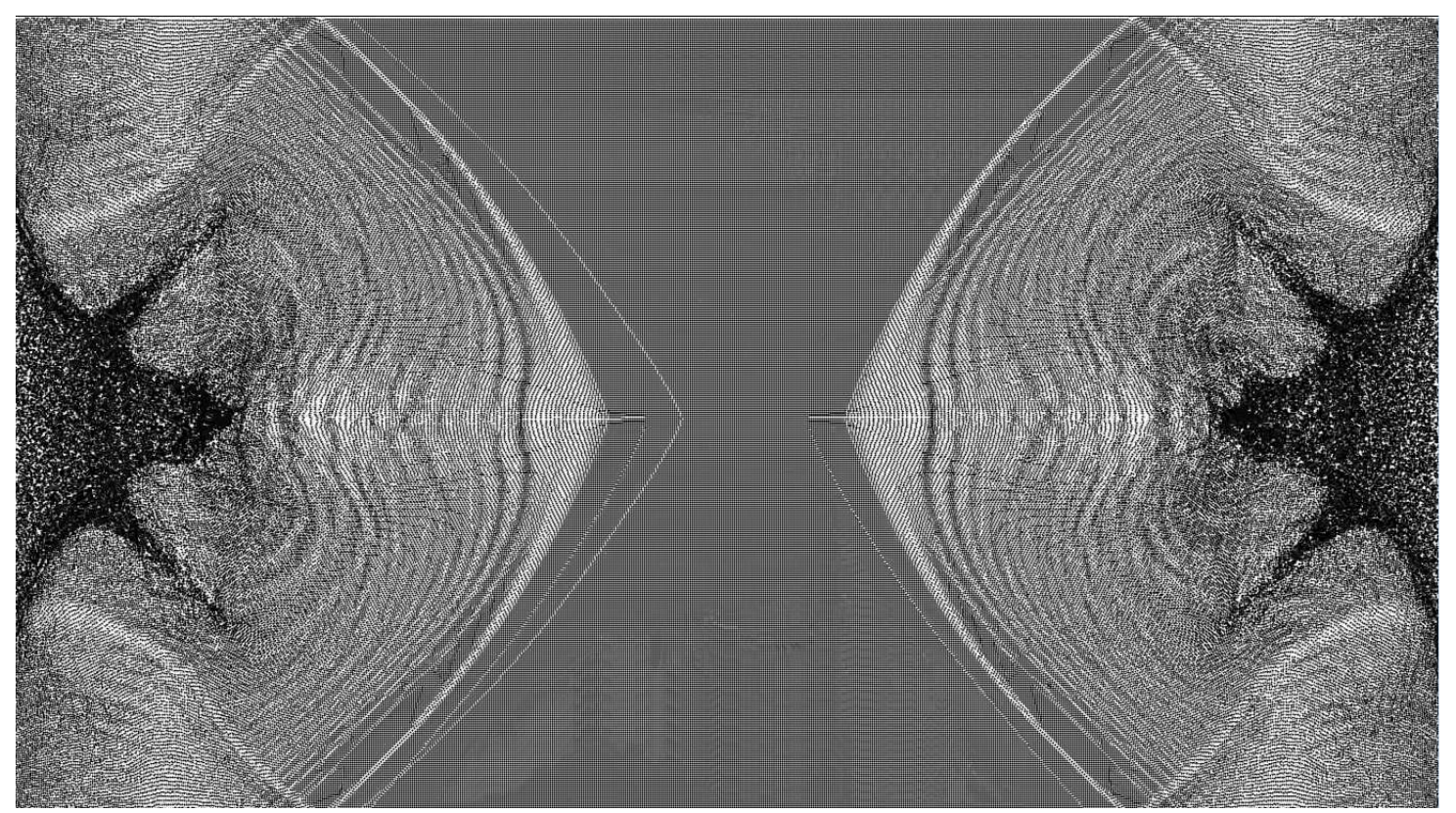

Figure 11: The wave fronts of the system are close to contact. The dark interference patterns are due to the reflections at the rectangular boundary.

Figure 12 shows the developmental phase where the wave fronts are already overlapping and a still undisturbed free wave interference pattern is created in the center of the array.

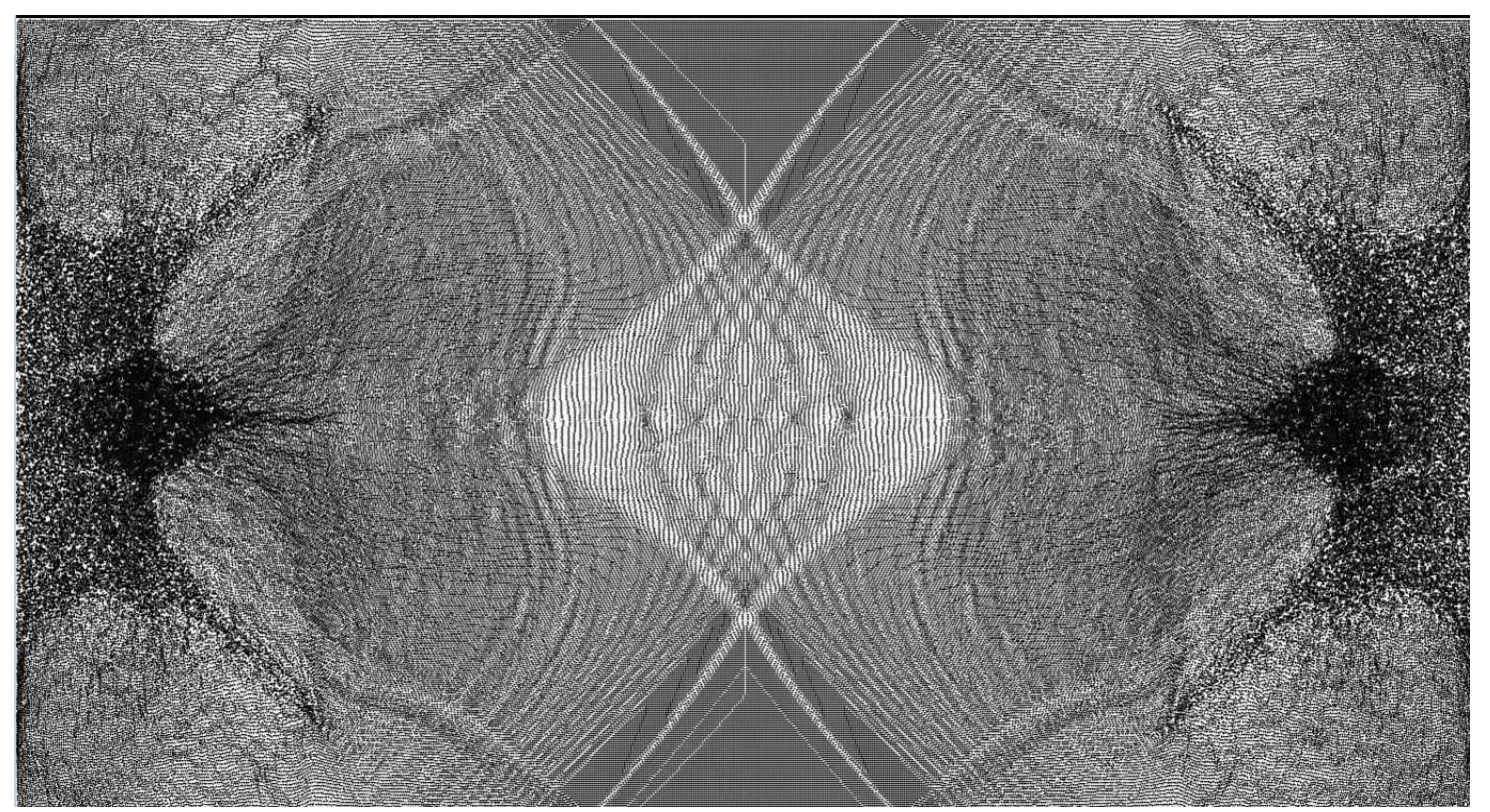

Figure 12: The wave fronts of the system are already overlapping and give rise to free wave interference effects.

In Figure 13 the central interference pattern has already almost been extinguished by the boundary reflections. The diagonal peaks of the dark interference patterns have reached the boundaries and bend away from them. 


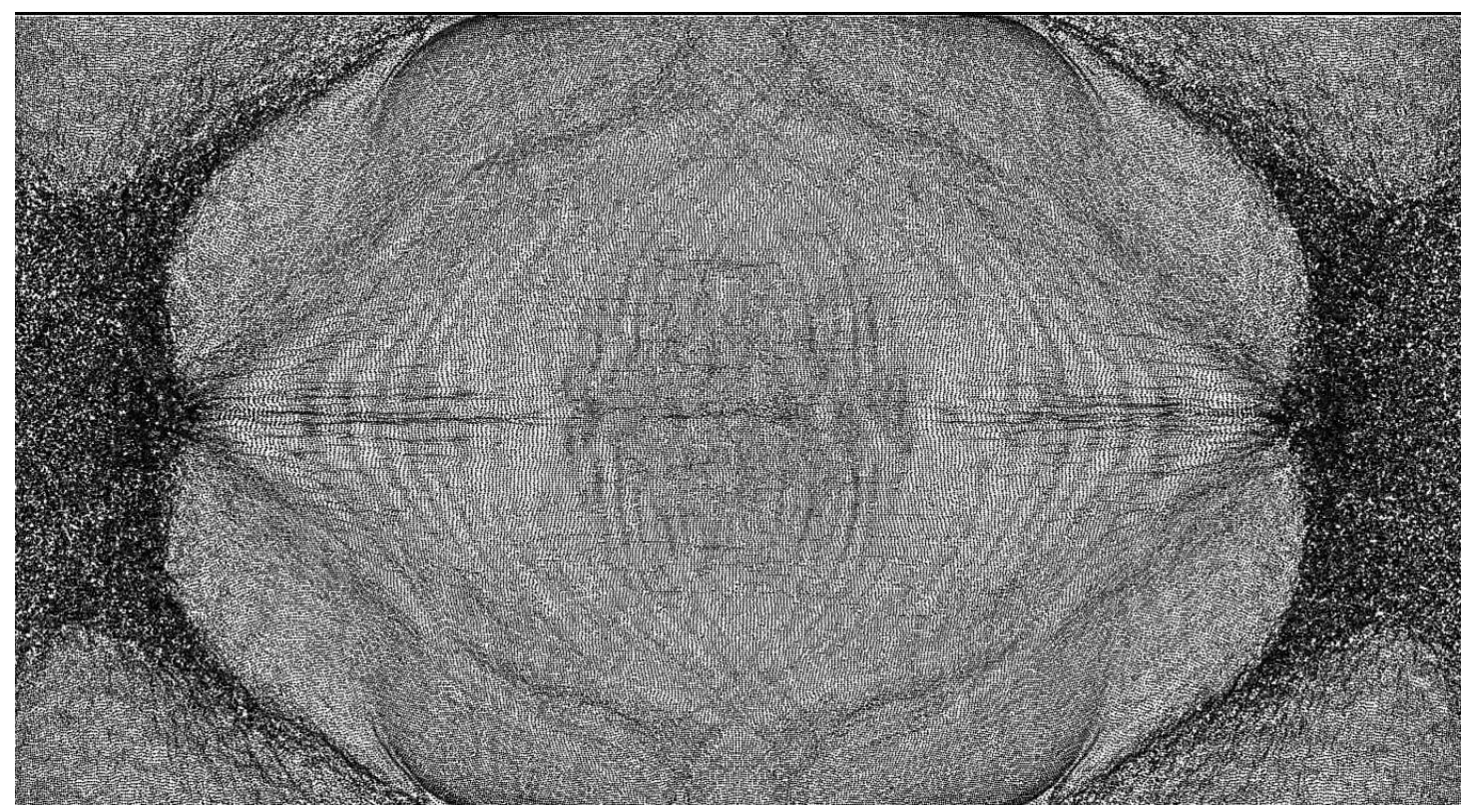

Figure 13: Free wave interference is superimposed by boundary reflections.

In Figure 14 the free wave interference pattern in the center of the array has vanished; there is only a faint horizontal line remaining that indicates the direction of wave propagation. The diagonal peaks of the dark interference patterns of the left and right boundary have converged and established an elliptic region.

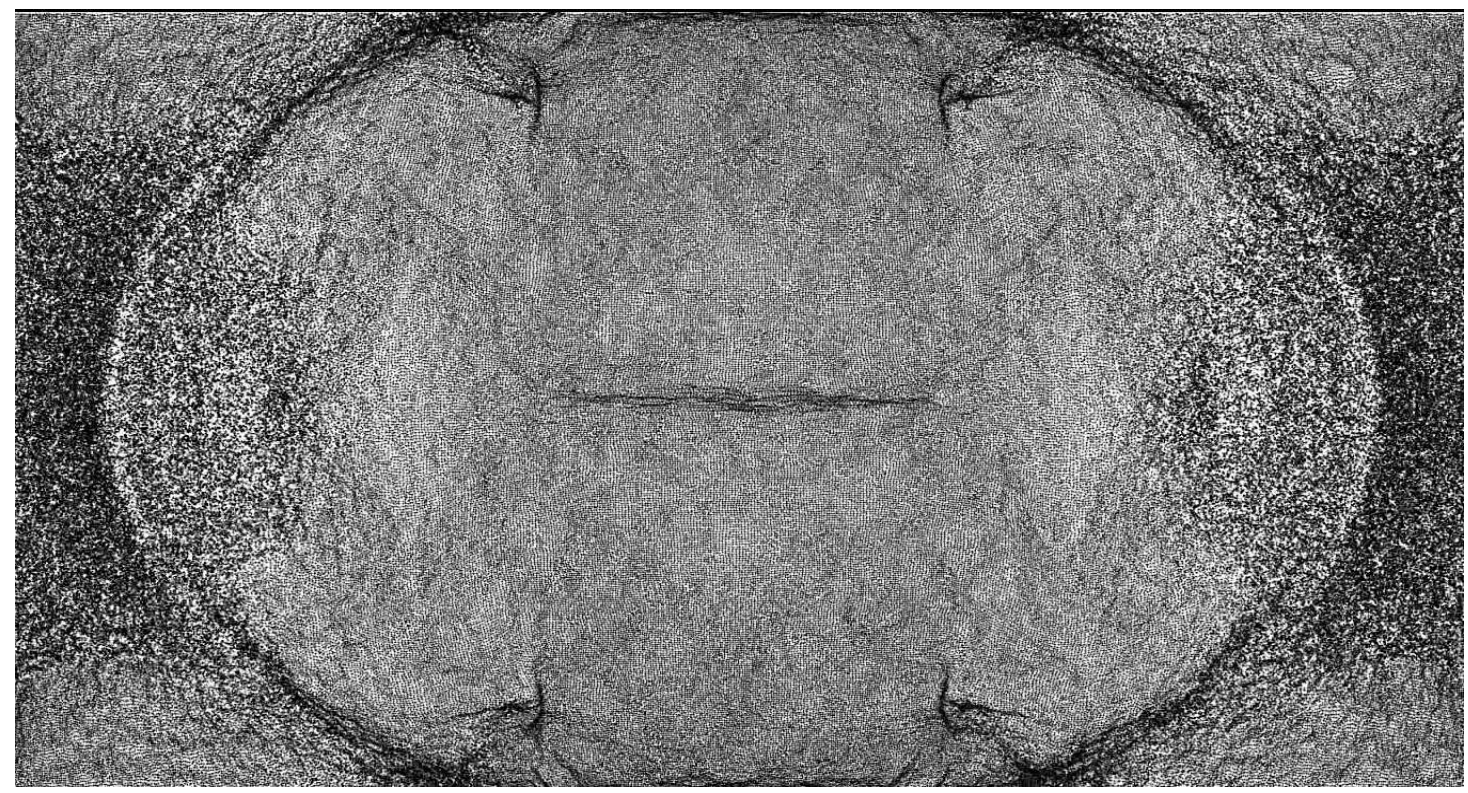

Figure 14: An almost elliptic interference pattern has formed that is caused by boundary shape and condition.

Figures 10 to 14 show only one of a multitude of wave and interference phenomena that can be produced by initial configurations and conditions set by the user. The key difference between a wave in some medium and a system of coupled oscillators is that wave phenomena are typically associated with propagation media which are modeled as continuous rather than discrete. In the close to continuum limit shown above, the array of oscillators can be viewed as a discrete model of a continuous material. 


\section{Summary and Conclusions}

A vibrating membrane can be regarded profitably as a continuous object, yet if one looks at a fine enough scale, the membrane is made up of atoms, suggesting a discrete model with a large number of elastically coupled particles. Due to a scale change that links the microstructure of the coupled particles with the macroscopic behavior of the membrane, the moving particles increasingly appear to the observer as a continuous system which includes wave behavior like interference effects.

With the intention that our students acquire insight into such a transition from microscopic interactions to macroscopic behavior of a complex system, an undergraduate student research project was initiated. Within our project-based learning framework, engineering students in their second semester of study have developed a computer program for the simulation of the dynamics of a two-dimensional mass-spring system. Three groups of three students worked simultaneously and competitively on the implementation of a numerical solution to this problem.

While the analytical and numerical solution of small coupled systems of linear differential equations is part of our standard Engineering Mathematics curriculum, the numerical solution of a larger system of differential equations can usually not be covered in a regular course. This software project, however, offered the opportunity to show our students the value of the just learned methods and algorithms, and was thus increasing their attentiveness and their appreciation for the recently learnt mathematical methods. The critical issue in teaching mathematics to engineering students is to find the right balance between practical applications of mathematical methods and in-depth understanding ${ }^{12}$.

The outcome of the project was rewarding with a convincing effort to performance ratio. The student-developed program enables the simulation of two-dimensional mass spring systems with an arbitrary number of particles with effortless ease. In addition, it allows a gradual approach to the system complexity necessary for the appearance of wave and interference phenomena.

Using this program within a traditional lecture brings a visual element into the teaching and learning process. The class can perform several initial and boundary condition scenarios and discuss the results. The dynamic visual output of the program can increase and enhance understanding of various wave phenomena; it provides a playful insight into the transition from discrete to continuous systems and is therefore well suited as a teaching aid.

The program can be downloaded, free of charge, from the website https://fahrzeugtechnik.fhjoanneum.at/software/CoupledOscillators/, and can be installed and executed on computers running the Windows and Linux operating systems. The authors would like to thank Wolfgang Dautermann for providing the compatibility layer for the program's execution on Linux operating systems. 


\section{Bibliography}

1. E. Kreyszig, "Advanced Engineering Mathematics”, John Wiley \& Sons, 9th Edition (2005)

2. J. T. Oden (Chair), "Simulation based engineering science—an NSF Blue Ribbon Report", retrieved 15 March 2016 from www.nsf.gov/pubs/reports/sbes_final_report.pdf (2006)

3. R. W. Batterman, "The Tyranny of Scales", in Robert W. Batterman, ed., The Oxford Handbook of Philosophy of Physics, Oxford University Press (2013)

4. J. Butterfield, "Less is different: Emergence and reduction reconciled", Foundations of Physics, 41(6), 1065-1135 (2011)

5. R. Feynman, R. Leighton, and M. Sands. The Feynman Lectures on Physics, Volume 2, Addison-Wesley, Reading, Massachusetts (1964)

6. G. Strang, "Introduction to Applied Mathematics", Wellesley-Cambridge Press (1986)

7. K. Slater and K. Gramoll, "Vibration Visualization using Longitudinal Vibration Simulator (LVS)", Proceedings of the ASEE Annual Conference and Exposition, Anaheim, CA (1995)

8. E. Bratschitsch, A. Casey, G. Bischof, and D. Rubeša, "3-phase multi subject project based learning as a didactical method in automotive engineering studies", Proceedings of the ASEE Annual Conference and Exposition, Honolulu, HI (2007)

9. G. Bischof, E. Bratschitsch, A. Casey, and D. Rubeša, "Facilitating engineering mathematics education by multidisciplinary projects", Proceedings of the ASEE Annual Conference and Exposition, Honolulu, HI (2007)

10. P. Hamill, “A Student's Guide to Lagrangians and Hamiltonians”, Cambridge University Press (2014)

11. C. G. Torre, "The Continuum Limit and the Wave Equation", Foundations of Wave Phenomena, 18 (2014)

12. S. S. Sazhin, “Teaching Mathematics to Engineering Students”, Int. J. Engng Ed. Vol. 14, No. 2 , 145 (1998) 